\title{
Midnight variations of spreading of ionospheric sporadic E-layers before earthquakes
}

\author{
Elena V. Liperovskaya ${ }^{1}$, Alexandra S. Silina ${ }^{1}$, Victor A. Liperovsky ${ }^{1}$, Claudia-Veronika Meister ${ }^{2,}$, \\ Dieter H.H. Hoffmann ${ }^{2}$, Pier Francesco Biagi ${ }^{3}$
}

\author{
${ }^{1}$ Institute of Physics of the Earth, Russian Academy of Sciences, Moscow, Russia \\ ${ }^{2}$ Institut für Kernphysik, Technische Universität Darmstadt, Darmstadt, Germany \\ ${ }^{3}$ Università di Bari, Dipartimento di Fisica, Bari, Italy
}

\author{
Article history \\ Received June 28, 2011; accepted February 12, 2012. \\ Subject classification: \\ Seismic risk, Statistical analysis, Earthquake precursors, Es-spread, Data analysis.
}

\begin{abstract}
In the present study, ionospheric phenomena caused by earthquakes of magnitudes $M>4.0$ were investigated. Night-time observations of the spreading of sporadic E-layers (Es-spread) performed every 15 min by the Dushanbe and Petropavlovsk-Kamchatsky (middle Asia) vertical sounding stations were studied. The mean relative occurrence frequency of Esspread at different values of the blanketing frequency $\mathrm{f}_{\mathrm{b}} \mathrm{E}_{\mathrm{s}}$ was considered, and the dependence of Es-spread on the season as well as on the year through an 11-yr solar activity cycle were studied. The $\mathrm{f}_{\mathrm{b}} \mathrm{E}_{\mathrm{s}}$ characterizes the maximum plasma density of the $\mathrm{E}_{\mathrm{s}}$-layer. The analysis shows that 13 days before seismic shocks in the Earth crust at depths of $\mathrm{h}<80 \mathrm{~km}$, the occurrence frequency of the Es-spread increases a few hours before midnight. This effect is characteristic of a strengthening of the turbulization of the E-layer plasma. On the basis that the radius of the earthquake preparation region $\left(\mathrm{R}_{\mathrm{D}}\right)$ is estimated by the Dobrovolsky formula $\mathrm{R}_{\mathrm{D}} \approx \exp (\mathrm{M}) \mathrm{km}$, it was found that Es-spread is observed more often when the distance between the epicenter and the radar station is not greater than $\mathrm{R}_{\mathrm{D}}+150 \mathrm{~km}$. In cases of earthquakes at greater distances and depths, no midnight effect was found. The authors act on the assumption that the Es-spread might be caused by acoustic waves with periods of $20 \mathrm{~s}$ to $5 \mathrm{~min}$. When such acoustic disturbances propagate from the Earth surface they will have maximum amplitudes if they move nearly vertically to greater altitudes.
\end{abstract}

\section{Introduction}

In a series of studies, the phenomenon of spreading of sporadic E-layers (Es-spread) has been studied in connection with earthquake preparation processes [Liperovsky et al. 2000, Liperovsky et al. 2008, Meister et al. 2011]. This phenomenon is seen as a diffusivity, or smearing, of traces of sporadic $E_{s}$-layers on ionograms of vertical sounding stations. The Es-spread effect apparently reflects the processes of large-scale (a few hundreds of meters) instabilities in the sporadic layers. This effect is a consequence of the turbulization of sporadic E-layers [Bowman 1985, Whitehead 1989]. Es-spread is a relatively rare phenomenon [Bowman 1985], and it can be mainly observed at night between 22:00 $\mathrm{h}$ and 03:00 $\mathrm{h}$ LT. The occurrence of sporadic E-layers depends on the season, as does the Es-spread effect. The effect is above all observed in summer, especially in the years of minimum activity of the solar cycle.

The first studies of seismo-ionospheric coupling were performed about 20 years ago for some strong earthquakes [Alimov 1989, Liperovsky et al. 1992]. These showed that the occurrence frequency (probability) of Es-spread increases during earthquake preparation times and immediately after the seismic shock, in comparison with all other times (the so-called "background periods"). Thus the idea arose that the occurrence frequency of Es-spread can be considered as an earthquake precursor. It had been suggested that acoustic, acoustic-gravity and electromagnetic disturbances that can occur during earthquake preparation processes might be important reasons for the turbulization of the ionosphere. In the ionosphere, turbulization that causes Es-spread should have scales of tenths of meters and tenths of kilometers, and the time scales are about a few tenths of minutes.

In more recent studies, the occurrence frequencies of Es-spread about 1-3 days before strong earthquakes in middle Asia (with magnitudes $M>5.5$ ) were investigated [Liperovsky et al. 2005, Silina et al. 2001]. These showed that the number of observed Es-spread effects increased over the distances between the epicenters of future earthquakes and the vertical sounding stations of up to $300 \mathrm{~km}$. The dependence of the Es-spread increase during earthquake preparation times in the day-time was not considered in these studies.

Liperovskaya et al. [2006] analyzed ionospheric effects 


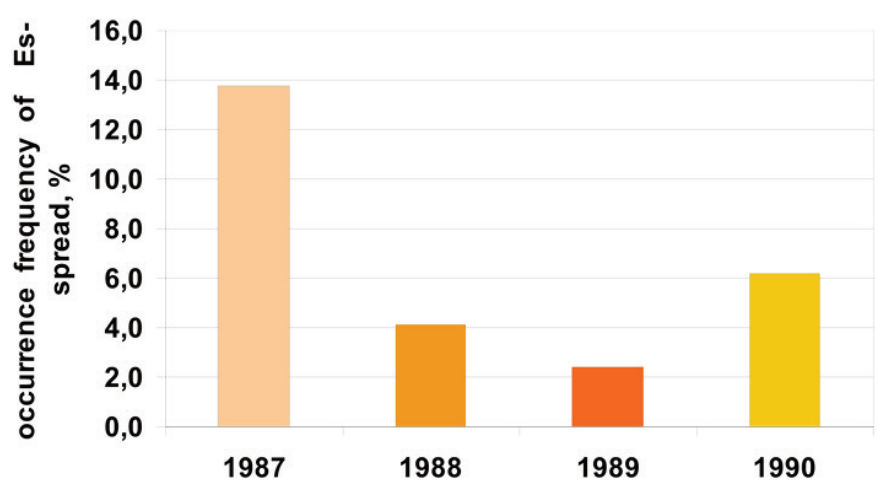

Figure 1. Occurrence frequency of the Es-spread observations as a function of the years from 1987 to 1990.

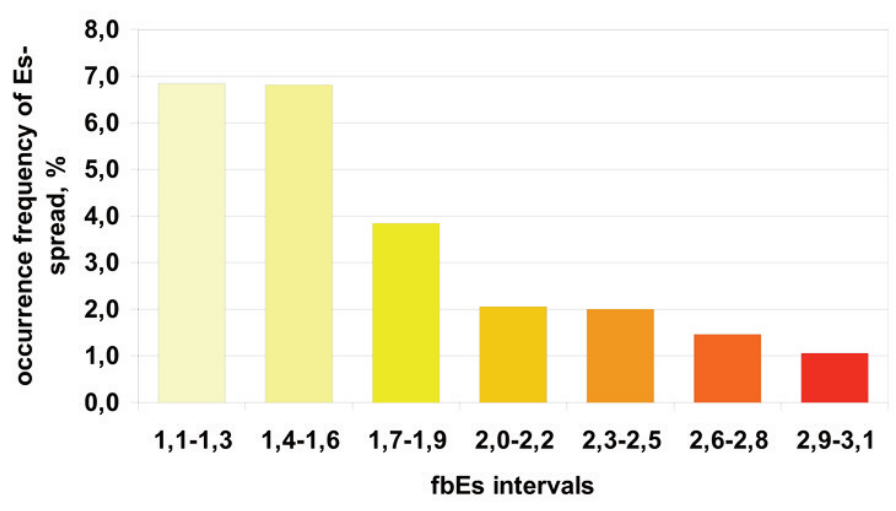

Figure 2. Occurrence frequency of the Es-spread observations as a function of the blanketing frequency $f_{b} E_{s}$.

from earthquakes with epicenters under the ocean in the environment of the vertical sounding station of Petropavlovsk-Kamchatsky. Taking into account earthquakes with magnitudes $M>4.0$ and sources at depths of up to 50 $\mathrm{km}$ (20 events), when compared with the background days, an increase in the occurrence frequency of Es-spread was found a few hours before midnight, 1-3 days before the earthquakes.

The main aim of the present study was to investigate the intensification of Es-spread in dependence on the daytime during periods of earthquake preparation using data from the vertical sounding station of Dushanbe, and to compare this with results obtained based on observations by the vertical sounding station of Petropavlovsk-Kamchatsky.

\section{Experimental data and results of the observations}

The present study analyzed Es-spread data that was observed by the ionospheric sounding station of Dushanbe $\left(\varphi=38.5^{\circ}, \lambda=68.8^{\circ}\right)$ every $15 \mathrm{~min}$ at night (between 16:00 and 04:00 LT) in the years 1987-1990. The Es-spread effect, as already indicated, is observed relatively rarely. For instance, in the year 1987, according to the data of the Dushanbe station, Es-spread, as the smearing of ionogram traces of sporadic Es-layers, was registered in about $14 \%$ of the Es-observations $(1,532$ cases out of 11,137$)$. These data were considered, which were related to a broad interval of $f_{b} E_{s}$ values of $1.3 \mathrm{MHz}$ to $3.1 \mathrm{MHz}$ (at higher frequencies, only a few individual Es-spread effects were found). During the increase in the solar activity, as the year of the activity maximum of the 11-yr solar cycle (1989) approached, the number of observed Es-spread phenomena decreased (Figure 1). This thus suggests that the possibility of a spread effect depends on the temperature of the neutral particles.

As was mentioned by Bowman [1985], the Es-spread effect was mainly obtained during times with smaller $f_{b} E_{s}$ values of $1 \mathrm{MHz}$ to $2 \mathrm{MHz}$. To give an idea of the numbers of spread effects in terms of the $f_{b} E_{s}$ values, the numbers of smearing effects in different frequency intervals of $f_{b} E_{s}$ (according to the data of the Dushanbe station) are related to the number of Es-layer recordings in this intervals (Figure 2). It follows from Figure 2 that the Es-spread depends on the maximum plasma density of the Es-layer (i.e. on $f_{b} E_{s}$ ). Generally, the Es-layer is thinner at smaller $f_{b} E_{s}$ values. Most often, Es-spread is observed at low $f_{b} E_{s}$ values, of $1.1 \mathrm{MHz}$ to $1.6 \mathrm{MHz}$.

Es-spread shows daily variation and strongly depends on the season. In Figures 3 and 4, the occurrence frequencies of the Es-spread in the years 1987-1990 are shown for winter and summer nights, as observed by the Dushanbe station. The relative occurrence frequency of the Es-spread in percent is the ratio of the number of Es-spread observations divided by the number of observations of the $E_{s}$-layer, times 100, in winter (Figure 3, October, November, December, January, February, March) and in summer (Figure 4, April, May, June, July, August, September). In summer, the number of observed Es-spread phenomena strongly increased after 23:00 LT. Large levels of Es-spread were observed until about 03:00 LT, and then the number of spread effects decreased strongly. During the winter, the number of Es-spread phenomena increased from about 22:00 LT, and decreased again at around 04:00 LT. This is the most dark time with the lowest temperatures of the day. The minimum of the temperature of the neutral particles was observed at E-layer altitudes in the night at about 03:00 LT.

In the present study, the daily behavior of the Es-spread occurrence on ionograms on seismoactive days was studied and compared with the Es-spread variations during background periods. In this process, based on the results of former studies [Liperovskaya et al. 2006, Liperovsky et al. 2000], the 1-3 days before a seismic shock, as days $(-2,-1,0)$, were considered to be the seismo-active times. All of the other days were interpreted as background days. Only earthquakes with magnitudes $M \geq 4$.0 were considered here. The analysis of these effects of such weak earthquakes was because sporadic layers are located at relatively small distances from the Earth surface (at altitudes of about $H \approx 100 \mathrm{~km}$ ), and seismo-ionospheric phenomena might thus also be caused by weak earthquakes. 


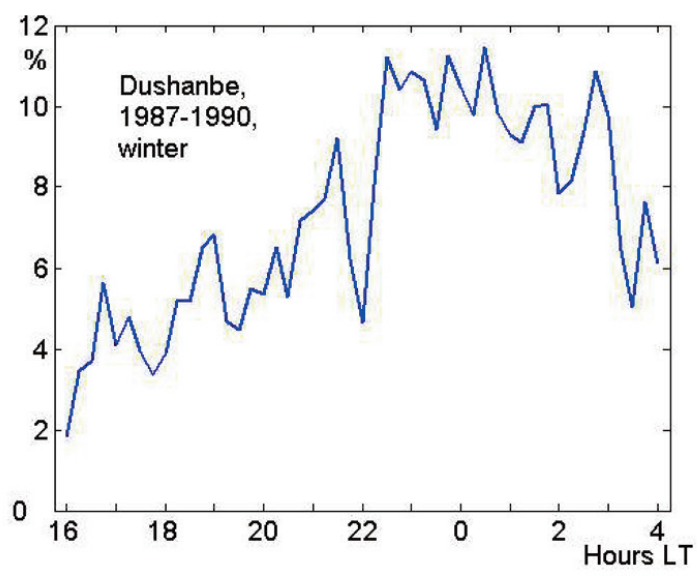

Figure 3. Relative occurrence frequency of the Es-spread observations in the evening hours and at night, as observed at the Dushanbe station in winter over the years of 1987-1990.

The radius of the earthquake preparation region $\left(R_{D}\right)$ can be estimated based on the Dobrovolsky formula, as $R_{D} \approx$ $\exp (M) \mathrm{km}$, where $M$ is the magnitude of the earthquake [Dobrovolsky et al. 1979]. It is clear from geometry considerations that weak disturbances that propagate upwards from the ground surface can change the properties of the ionosphere if the dimension $d$ of the preparation region is of the order of the distance between the Earth surface and the E layer, i.e. $H \approx d=2 R_{D}$. Consequently, the earthquake magnitudes should be larger than $4, \exp (4) \approx 55 \mathrm{~km}$.

We suggest that the Es scattering in the E-layer (i.e. the Es-spread on the ionograms) can be caused by acoustic waves with periods of $20 \mathrm{~s}$ to $5 \mathrm{~min}$. The acoustic waves will attain maximum amplitude if they propagate almost perpendicularly to the Earth surface. According to previous results, a variation in the occurrence of Es-spread was observed for earthquakes with depths $h<50 \mathrm{~km}$, the epicenters of which are situated at distances $R<R_{D}+100$ $\mathrm{km}$. Taking these suggestions and results into account, in the earthquakes investigated in the present study, their preparation regions were located relatively near to the ionosondes, $R<R_{D}+150 \mathrm{~km}$.

Here, observations of the station Dushanbe are considered. In doing so, the three nights $(-2,-1,0)$ before the earthquakes were also considered as seismo-active times. The other nights are interpreted as the background period. Only data recorded every $15 \mathrm{~min}$ between 16:00 and 04:00 LT by vertical sounding are taken into account here. Furthermore, the numbers of Es-spread observations were calculated for all 49 points in time, separately for seismoactive and background periods (of the year 1987, or of the time period 1988-1990). In the year 1987, the Es-spread effects were observed much more often than in the years 1988-1990. In principle, in the present study, the method of superposition of epochs is used. Here, the homogeneity of the data is of great importance for the determination of the weak seismo-ionospheric effects. Therefore, the data of the year 1987 and the years 1988-1990 were analyzed separately.

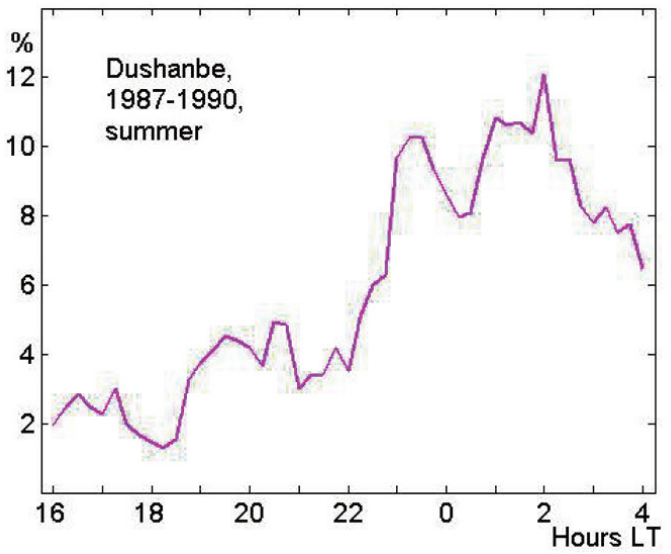

Figure 4. Relative occurrence frequency of the Es-spread observations in the evening hours and at night, as observed by the Dushanbe station in summer over the years of 1987-1990.

Also, for the study, it was useful to divide the whole set of experimental data into two independent blocks, and to analyze the seismo-ionospheric effects of each of these. This approach allowed conclusions to be made on the daytime of the increase of the observed Es-spread effects that were connected with earthquake preparation processes.

It is shown here that 1-3 days before earthquakes, in the surroundings of Dushanbe there was also a growth in Esspread. Figures 5 and 6 show the results of the analysis for the relative occurrence frequency of the Es-spread obtained for the data of the Dushanbe station. Only earthquakes with depths $<80 \mathrm{~km}$ were considered. The method of superposition of epochs was separately applied for the time intervals of 1987 (Figure 5) and 1988-1990 (Figure 6). The division of the data into these two time intervals of 1987 and 1988-1990 was chosen in such a way that there were sufficient data for in-depth analyses and conclusions. In Figures 5 and 6, the number of measured Es-spread effects is shown as a function of the local time (LT) for the seismo-active nights

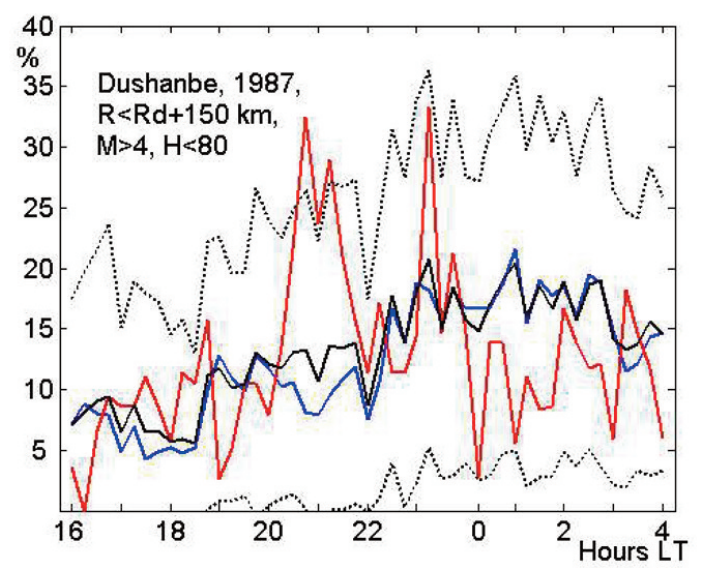

Figure 5. Relative occurrence frequency of the Es-spread observations during seismo-active (red line) and background (blue line) days in the evening and around midnight, as a function of the local time (LT). The results were obtained using the method of superposition of epochs for the data obtained by the Dushanbe station in 1987. Black line, mean obtained with the Monte-Carlo method; dotted lines, \pm 2 standard deviation intervals. 


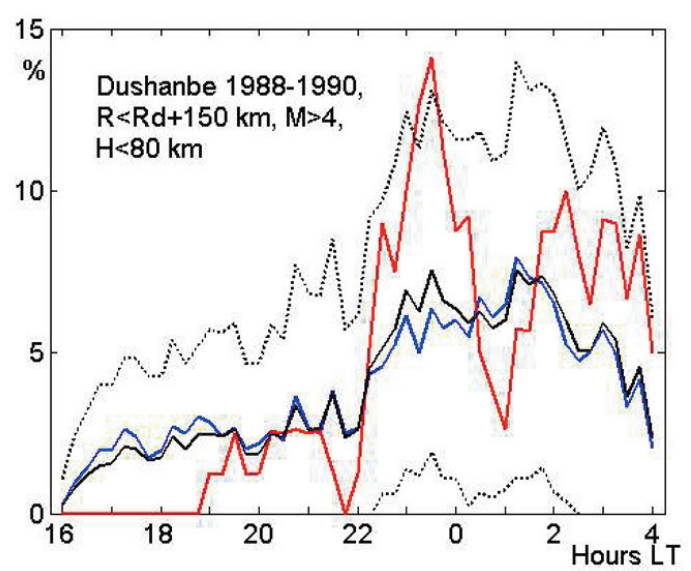

Figure 6. Relative occurrence frequency of the Es-spread observations during seismo-active (red line) and background (blue line) days in the evening and around midnight, as a function of the local time (LT). The results were obtained using the method of superposition of epochs for the data obtained by the Dushanbe station from 1988 to 1990 . Black line, mean obtained with the Monte-Carlo method; dotted lines, \pm 2 standard deviation intervals.

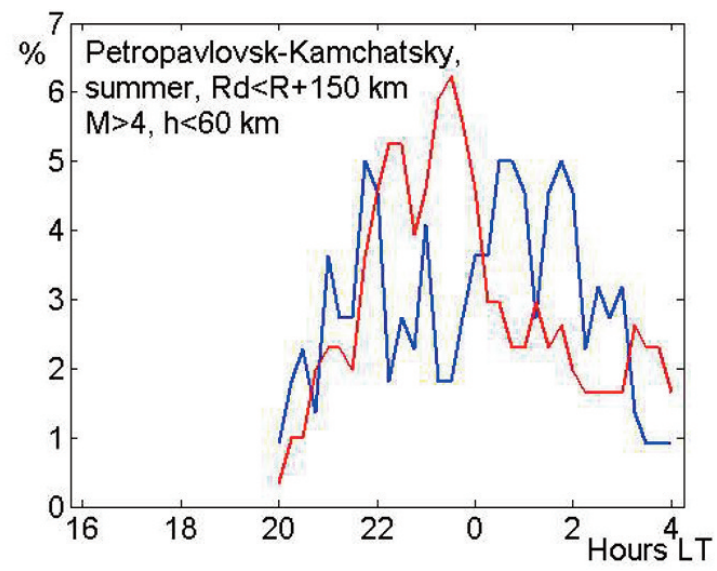

Figure 7. Relative occurrence frequency of the Es-spread observations during seismo-active (red line) and background (blue line) days in the evening and around midnight, as a function of the local time (LT). The results were obtained using the method of superposition of epochs for data obtained by the Petropavlovsk-Kamchatsky station in 2004 [from Liperovskaya et al. 2006].

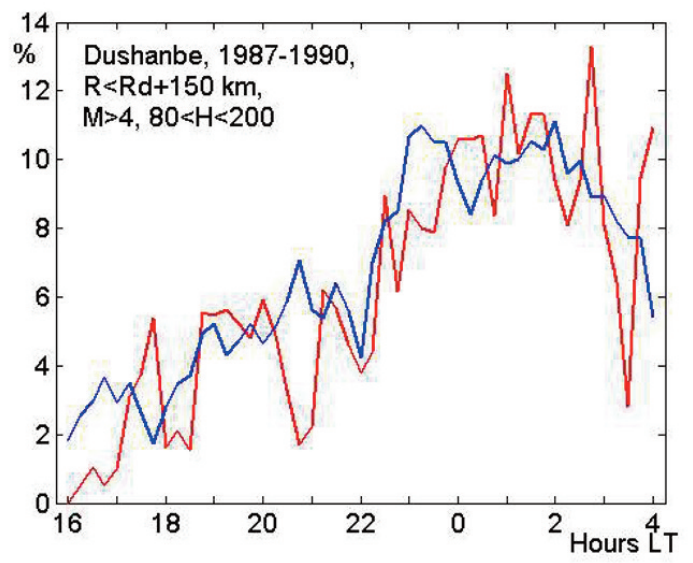

Figure 8. Relative occurrence frequency of the Es-spread observations during seismo-active (red line) and background (blue line) days in the evening and around midnight, as a function of the local time (LT). The results are shown for earthquakes with epicenters in depths $>80 \mathrm{~km}$. (red line) and the background nights (blue line), separately. The black line shows the mean value obtained with the Monte-Carlo method, and the dotted lines indicate the \pm 2 standard deviation intervals. The y-axes of the figures show the Es-spread data at times of multiples of $15 \mathrm{~min}$. The results were normalized to the sum of all of the observations between 16:00 and 04:00 LT.

For every time interval (1987 and 1988-1990), 300 series of virtual earthquakes were constructed using the Monte Carlo method. The number of virtual earthquakes in any series is equal to the number of real earthquakes. The method of superpositions of time intervals in the vicinity of earthquakes was applied to determine for every series the day-time dependence of the Es-spread occurrence frequency. Thus, for any time interval, 300 versions of the day-time dependence were found. For these 300 versions, the mean values and the mean standard deviations were calculated. The mean values differed only slightly from the background values (Figures 5 and 6, black and blue curves). The standard deviations depend on the number of earthquakes within a series. In 1987, 18 earthquakes occurred. The number of earthquakes during the time period 1988-1990 was 48 .

Figures 5 and 6 show that there was a growth in the number of Es-spread effects 1-3 days before earthquakes, over a few hours before midnight in both 1987 and for the time interval of 1988-1990. An additional analogous analysis of the data of the Petropavlovsk-Kamchatsky vertical sounding station confirms these results (see Figure 7, taken from Liperovskaya et al. [2006]).

Furthermore, using the data of the Dushanbe station, we investigated at what distance between the earthquakes and station, and at what depths of the seismic shock, the increase of the Es-spread obtained was seen to disappear. Therefore, the method of superposition of epochs was applied to earthquakes with magnitudes $M>4$ and depths $80<h<200 \mathrm{~km}$ : these occurred at distances $R<\exp (M)+$ $150 \mathrm{~km}$ from the vertical sounding station. The result of this is that no growth in the number of Es-spread effects occurs in the hours before midnight (Figure 8).

Similarly, the spatial scales of the observed increases before midnight were investigated for earthquakes with magnitudes $M>4$ and depths $h<80 \mathrm{~km}$, which appeared at distances $\exp (M)+150<R<\exp (M)+300 \mathrm{~km}$ from the station. Again, no effects were found (Figure 9).

\section{Discussion and conclusions}

The analysis of Es-spread data observed by the Dushanbe vertical sounding station shows that 1-3 days before earthquakes in the Earth crust at depths $h<80 \mathrm{~km}$ the number of the spread effects increases a few hours before midnight. This phenomenon is characteristic of an additional turbulization of the ionospheric E-layer. It was found that the growth of the number of Es-spread effects occurs for 


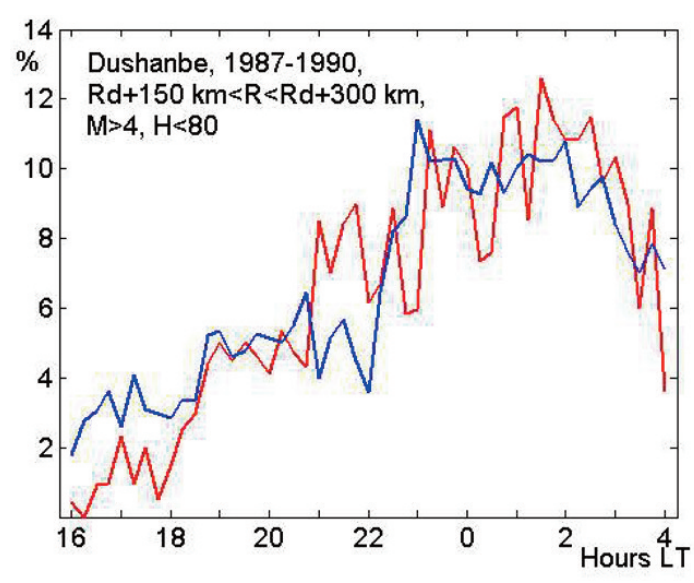

Figure 9. Relative occurrence frequency of the Es-spread observations during seismo-active (red line) and background (blue line) days in the evening and around midnight, as a function of the local time (LT). The results are shown for earthquakes with epicenters at distances from the vertical sounding station $>(\exp (M)+150) \mathrm{km}$.

earthquakes for which the magnitude $M$ and the distance between the epicenter and vertical sounding station satisfy the relation $R<\exp (M)+150 \mathrm{~km}$ for $M>4$. For seismic shocks at greater distances from the station, and for shocks with deeper sources, no effects were found.

For the possible physical mechanisms of these described phenomena, it can be concluded that the Es-spread is mainly caused by acoustic disturbances of the neutral atmosphere, with periods of $20 \mathrm{~s}$ to $100 \mathrm{~s}$ [see e.g. Liperovskaya et al. 2011, Meister et al. 2011]. These disturbances propagate up into the atmosphere from the Earth surface within the transparency interval of the waves [Blanc 1985, Molchanov et al. 2003].

An simultaneous increases in the Es-spread occurrence frequency and decreases in the amplitudes of the ultra-low frequency variations of the magnetic field in the frequency interval of $0.005 \mathrm{~Hz}$ to $0.2 \mathrm{~Hz}$ have been observed at Kamchatka before a few earthquakes [Molchanov et al. 2003, Molchanov et al. 2005], Molchanov et al. [2003, 2005] have suggested that the absorption of the magnetic field pulses increases in the turbulized atmosphere, and thus the amplitudes of the ultra-low frequency disturbances decrease.

\section{References}

Alimov, O.A., M.B. Gokhberg, E.V. Liperovskaya, I.L. Gufeld, V.A. Liperovsky and L.N. Roubtsov (1989). Anomalous characteristics of the middle latitude $E_{s}$ layer before earthquakes, Phys. Earth Planet. Inter., 57, 76-81.

Blanc, E. (1985). Observations in the upper atmosphere of infrasonic waves from natural or artificial sources: A summary, Ann. Geophys., 3 (6), 673-688.

Bowman, G.G. (1985). Some aspects of middle-latitude spread Es and its relationship with spread F, Planet. Space Sci., 33 (9), 1081-1089.

Dobrovolsky, I.R., S.I. Zubkov and V.I. Myachkin (1979). Estimation of the size of earthquake preparation zones, Pure Appl. Geophys., 117, 1025-1044.
Liperovskaya, E.V., C.-V. Meister, O.A. Pokhotelov, M. Parrot, V.V. Bogdanov and N.E. Vasil'eva (2006). On Esspread effects in the ionosphere connected to earthquakes, Nat. Hazards Earth Syst. Sci., 6, 740-744.

Liperovskaya, E.V., V.V. Bogdanov, P.F. Biagi, C.-V. Meister and V.A. Liperovsky (2011). Daytime variations of foE connected to earthquakes, Nat. Hazards Earth Syst. Sci., 11, 1807-1812; doi:10.5194/nhess-11-1-2011.

Liperovsky, V.A., O.A. Pokhotelov and S.L. Shalimov (1992). Ionospheric precursors of earthquakes, Moscow, Nauka.

Liperovsky, V.A., O.A. Pokhotelov, E.V. Liperovskaya, M. Parrot, C.-V. Meister and A. Alimov (2000) Modification of sporadic E-layers caused by seismic activity, Surveys Geophys., 21, 449-486.

Liperovsky, V.A., C.-V. Meister, E.V. Liperovskaya, N.E. Vasil'eva and O. Alimov (2005). On spread- $E_{s}$ effects in the ionosphere before earthquakes, Nat. Hazards Earth Syst. Sci., 5, 59-62.

Liperovsky, V.A., O.A. Pokhotelov, C.-V. Meister and E.V. Liperovskaya (2008). Physical models of coupling in the lithosphere-atmosphere-ionosphere system before earthquakes, Geomagn. Aeron., 48 (6), 795-806.

Meister, C.-V., B. Mayer, P. Dziendziel, F. Fülbert, D.H.H. Hoffmann and V.A. Liperovsky (2011). On the acoustic model of lithosphere-atmosphere-ionosphere coupling before earthquakes, Nat. Hazards Earth Syst. Sci., 11, 1011-1017; doi:10.5194/nhess-11-2011.

Molchanov, O., A. Schekotov, E. Fedorov, G. Belyaev and E. Gordeev (2003). Preseismic ULF electromagnetic effect from observation at Kamchatka, Nat. Hazards Earth Syst. Sci., 3, 203-209.

Molchanov, O., A. Schekotov, M. Solovieva, E. Fedorov, V. Gladyshev, E. Gordeev, V. Chebrov, D. Saltykov, V.L. Sinitsin, K. Hattory and M. Hayakawa (2005). Near-seismic effects in ULF fields and seismo-acoustic emission: Statistics and explanation, Nat. Hazards Earth Syst. Sci., 5, 1-10.

Silina, A.S., E.V. Liperovskaya, V.A. Liperovsky and C.-V. Meister (2001). Ionsospheric phenomena before strong earthquakes, Nat. Hazards Earth Syst. Sci., 1, 113-118.

Whitehead, J.D. (1989). Recent work on mid-latitude and equatorial sporadic-E, J. Atmos. Terr. Phys., 51 (5), 401424.

\footnotetext{
${ }^{\star}$ Corresponding author: Claudia-Veronika Meister, Institut für Kernphysik, Technische Universität Darmstadt, Darmstadt, Germany; email: c.v.meister@skmail.ikp.phyik.tu-darmstadt.de.
}

(C) 2012 by the Istituto Nazionale di Geofisica e Vulcanologia. All rights reserved. 